\title{
Effect of Cryotherapy On Pain Scores and Satisfaction Levels of Patients in Cataract Surgery Under Topical Anesthesia
}

Marzieh Beigom Khezri ( $\nabla$ mkhezri88@gmail.com )

Qazvin University of Medical Sciences

Abbas Akrami

Qazvin University of Medical Sciences

Matina Majdi

Qazvin University of Medical Sciences

Bijan Gahandideh

Qazvin University of Medical Sciences

Navid mohammadi

Qazvin University of Medical Sciences

\section{Research}

Keywords: Cryotherapy, phacoemulsification, topical anesthesia, cataract

Posted Date: November 11th, 2021

DOl: https://doi.org/10.21203/rs.3.rs-966789/v1

License: (c) (1) This work is licensed under a Creative Commons Attribution 4.0 International License. Read Full License 


\section{Abstract \\ Background}

To evaluate the effects of cryotherapy on pain scores and satisfaction levels of patients during cataract surgery under topical anesthesia.

\section{Methods}

Eighty patients aged between 55 and 75 years scheduled for cataract surgery were randomly allocated to two study groups to receive topical anesthesia with cryotherapy (TC) or topical anesthesia alone (T) groups. Visual analog pain scores, patient satisfaction level, hemodynamic parameters, and quality of operating conditions were recorded.

\section{Results}

Cryotherapy significantly reduced VAS pain scores during surgery $(P=0.014)$. Although no significant difference in postoperative pain scores, opioid consumption, heart rate, and mean arterial blood pressure was seen in the postoperative period. The surgeon reported better quality of operating conditions in the TC group $(P=0.018)$.

\section{Conclusion}

Cryotherapy as a complementary method with topical anesthesia reduced pain scores of patients during surgery. It also produced a better quality of operating conditions for surgeons. There was no significant difference in either postoperative pain scores or opioid consumption.

\section{Introduction}

Cataract surgery is one of the most common surgical procedures in men and women aged between 50 to 80. [1] The most common method for cataract surgery is phacoemulsification with topical anesthesia. ${ }^{[2]}$ Topical anesthesia protects patients from the possibility of globe perforations, optic nerve injury, and risk of respiratory arrest. ${ }^{[2,3]}$ Nowadays, systemic analgesic and hypnotic agents are used to relieve patient distress and enhance the patient's satisfaction with surgery. ${ }^{[3]}$ However, these agents may cause disorientation, respiratory depression, hypotension, bradycardia, and cardiovascular depression. Furthermore, each of these agents may lead to impairment of the patient's cooperation during surgery and work less than ideal agents for the management of conscious sedation. ${ }^{[1-3]}$ Therefore, the potential clinical benefits of the new approach in this setting need to be assessed. 
The analgesic role of low temperatures during cataract surgery, even without the association of topical anesthetics was described. ${ }^{[4,5]}$ The possible action mechanism of cryoanalgesia in reducing pain is related to a decrease in the activities in the polymodal sensitive neurons. ${ }^{[6,7]}$ Thus, during phacoemulsification with cold intraocular irrigation solution, the cold would reduce the transmission of painful mechanical stimulus. ${ }^{[4,5]}$ On the other hand, another study reported a dubious benefit of cryoanalgesia in phacoemulsification, but no difference in the severity of pain during phacoemulsification with topical anesthesia either with or without cryoanalgesia. [8]

Due to the controversial reports about the analgesic effects of cryotherapy during cataract surgery, the present study was conducted to compare patient-reported pain intensity during cataract surgery with topical anesthesia versus topical combined to cryotherapy.

\section{Methods}

In a prospective, double-blinded randomized trial, 80 patients were recruited for cataract surgery by phacoemulsification after the approval of the Institutional Ethics Committee and written informed consent. The consolidated standards of reporting trials (CONSORT) recommendations for reporting randomized controlled clinical trials were followed [Figure 1]. Inclusion criteria were patients aged 55-75 years scheduled for elective cataract surgery with intraocular lens implantation using phacoemulsification under topical anesthesia for the first time according to the American Society of Anesthesiologists (ASA) physical status classification I-III. Exclusion criteria were patients with sleep disorders, depressive disorder, or expected compliance problems (known psychiatric disease), epilepsy, insufficient pupillary dilation, nystagmus, deafness, ongoing treatment with hypnotics or psychotropic drugs (including opioids) within a week before admission, daily analgesic NSAIDs treatment. All patients received $3 \mathrm{mg}$ of melatonin one hour before entering the operating room to reduce anxiety.

Eighty patients aged between 55 and 75 scheduled for cataract surgery were randomly allocated to two study groups to receive either topical anesthesia with cryotherapy (TC) or topical anesthesia alone (T) using a computer-generated randomization schedule. All solutions to be instilled during the operation, except the povidone drops, were cooled to around 4C. Mydriasis was achieved by tropicamide 1\%. No patient received preoperative sedation. An eye mask of cold gel (Eyes Pack Single) was placed over the eye for about 10 minutes before surgery. After washing the periocular skin with chlorhexidine $0.5 \%$, ocular asepsis was achieved using povidone $5 \%$ drops. The intraocular irrigation was performed by a solution at room temperature (approximately 23C) in group T and $4 \mathrm{C}$ in group TC. The solution for group TC was kept in a refrigerator at approximately $4 \mathrm{C}$ temperature. The storage advised by the manufacturer was $2 \mathrm{C}$ to $27 \mathrm{C}$. The same surgeon performed all surgeries. The lens opacity was almost the same in both groups, according to the LOCS.

Phacoemulsification was performed through a temporal clear corneal incision technique. An applicator was used to hold the globe to avoid touching the conjunctiva or sclera. In group TC, during 
phacoemulsification, the cornea was kept chilled with cold BSS. The lens nucleus was hydro-dissected with cold BSS irrigation.

No other sedative or analgesic agent was used. At the preoperative visit, the verbal pain scores (VPS) ranged from 0 to $10(0=$ no pain and $10=$ worst pain imaginable). Patients were monitored by an electrocardiogram, noninvasive measurement of blood pressure, and pulse oximetry (SPO2). Pain scores were recorded for each patient several times: before premedication ( $\mathrm{T} 1$ ), 60 minutes after premedication, on arrival at the operating room (T2), during the operation period (T3), and also postoperatively before discharge from the recovery room (T4). At the end of the surgery, the patients were asked about the severity of their pain during the operation period according to the VPS criteria explained before premedication.

After surgery, the sedation level of patients was measured according to the modified Ramsay score scale using a 3-point scale with 1 = anxious, 2 = calm and oriented, $3=$ calm and drowsiness.

Complications such as respiratory depression, headache, nausea, vomiting, and chills were also recorded.

Postoperatively, the operating surgeon, who was unaware of patient assignment, was asked to assess the adequacy of intraoperative conditions according to the following scale: excellent (complete calmness and cooperating with the surgeon), good (slight undesirable movements of the eye, and poor (severe undesirable movements of the eye and un-cooperating). Patient satisfaction was also assessed by the lowa Satisfaction with Anesthesia Scale (ISAS). ${ }^{[9,10]}$

According to a previously published study. ${ }^{[8]}$, considering a significance level of $5 \%$ and power of $80 \%, 50$ patients should be included (25 per group) to the minimum difference between groups become significant. Parametric data were expressed as the mean \pm SD. The normality of the distribution was tested by a one-sample Kolmogorov-Smirnov test. The $t$-test analysis was used for continuous parametric variables. Nonparametric data were expressed as the median interquartile range. The pain scores and patient satisfaction were compared between groups by Fisher's exact test; otherwise, the Mann-Whitney $U$ test was used. A $P$ value $<0.05$ was considered significant. Statistical analysis was carried out using SPSS version 16 for Windows (SPSS, Chicago, IL).

\section{Results}

We excluded ten out of 90 patients due to logistical issues or other violations of the study protocol. Eighty patients were randomly assigned to two groups [Fig. 1]. There were no significant differences between the two groups regarding the demographic features including age, gender, and duration of surgery [Table 1]. As shown in Table 2, the intensity of pain scores in the patients in the cryotherapy (TC) group was significantly lower than the control group ( $p=0.014)$. In other words, $67 \%$ of patients in the TC group did not report any pain, while $42.5 \%$ of T group patients had no pain. Patients' satisfaction was compared in two groups by the lowa questionnaire. This questionnaire had 11 questions; each question had six levels of answers so, the minimum and maximum scores were 11 and 66 . The difference of these values 
between the TC $(88 / 0 \pm 48)$ and T $(4 / 1 \pm 49)$ groups was insignificant $(p=0.5)$. As shown in Table 3 , the surgeon described his satisfaction level as "excellent" in $20 \%$ and $5 \%$ of the patients of TC and T groups, respectively. At the same time, he mentioned a "bad" experience in $5 \%$ and $27.5 \%$ of the TC and T groups. The level of surgeon satisfaction in the TC group was significantly different from the T group $(p=0.01)$.

Table 1

Demographic data

\begin{tabular}{|c|c|c|c|}
\hline Variable & $\begin{array}{l}\text { T group } \\
(n=40)\end{array}$ & $\begin{array}{l}\text { TC group } \\
(n=40)\end{array}$ & P-value ${ }^{\rrbracket}$ \\
\hline Age & $2 / 6 \pm 9 / 65$ & $9 / 6 \pm 1 / 66$ & $5 / 0$ \\
\hline Gender** & $(5 / 57) 23$ & $(5 / 42) 17$ & $13 / 0$ \\
\hline Duration of surgery* & $87 / 1 \pm 3 / 11$ & $07 / 2 \pm 8 / 10$ & $239 / 0$ \\
\hline \multicolumn{4}{|c|}{${ }^{*}$ Data are presented as mean \pm SD. Independent samples t-test was used } \\
\hline \multicolumn{4}{|c|}{ 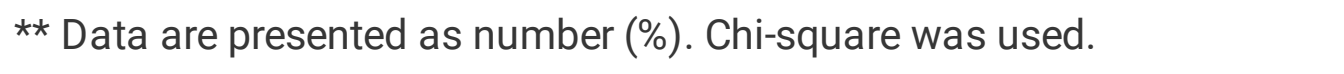 } \\
\hline \multicolumn{4}{|c|}{ T group =Patients received topical anesthesia. } \\
\hline TC group= Patient & d topical a & a - crayot & \\
\hline
\end{tabular}

Table 2

The relative frequency of pain intensity in two groups

\begin{tabular}{|c|c|c|c|}
\hline Variable & $\begin{array}{l}\text { T group } \\
(n=40)\end{array}$ & $\begin{array}{l}\text { TC group } \\
(n=40)\end{array}$ & P-value ${ }^{\rrbracket}$ \\
\hline No pain & $(5 / 42) 17$ & $(5 / 67) 27$ & \multirow[t]{4}{*}{$014 / 0$} \\
\hline Mild pain & $(5 / 32) 13$ & (30) 12 & \\
\hline Moderate pain & (20) 8 & (0) 0 & \\
\hline Sever pain & (5) 2 & $(5 / 2) 1$ & \\
\hline \multicolumn{4}{|c|}{${ }^{\circledR}$ Data are presented as number (\%). Chi-square was used. } \\
\hline \multicolumn{4}{|c|}{ T group =Patients received topical anesthesia. } \\
\hline \multicolumn{4}{|c|}{ TC group= Patients received topical anesthesia - crayotherap } \\
\hline
\end{tabular}


Table 3

Comparison of surgeon satisfaction in two groups

\begin{tabular}{|llll|}
\hline Variable & $\begin{array}{l}\text { T group } \\
(\mathbf{n}=\mathbf{4 0})\end{array}$ & $\begin{array}{l}\text { TC group } \\
(\mathbf{n}=\mathbf{4 0})\end{array}$ & P-value \\
\hline Excellent & $(5) 2$ & $(20) 8$ & $01 / 0$ \\
\hline Good & $(5 / 57) 23$ & $(65) 26$ & - \\
\hline Moderate & $(10) 4$ & $(10) 4$ & - \\
\hline Bad & $(5 / 27) 11$ & $(5) 2$ & - \\
\hline *Data presented as a number (percentage). \\
\hline \begin{tabular}{ll}
\hline Chi-square test was used. \\
\hline T group =Patients received topical anesthesia.
\end{tabular} \\
\hline
\end{tabular}

Comparison of sedation score values (Table 4) in cryotherapy and control groups showed no significant difference $(p=0.87)$.

Table 4

Distribution of the relative frequency of sedation level according to Ramsay score.

\begin{tabular}{|c|c|c|c|}
\hline Variable* & T group $(n=40)$ & TC group $(n=40)$ & P-value ${ }^{\bigotimes}$ \\
\hline Head and neck movement, & (20) 8 & $(5 / 17) 7$ & \multirow[t]{4}{*}{$879 / 0$} \\
\hline Mild hand movements, & $(5 / 17) 7$ & $(5 / 22) 9$ & \\
\hline Patients groaning, & $(5 / 17) 7$ & $(5 / 12) 5$ & \\
\hline Complete relaxation. & (45) 18 & $(5 / 47) 19$ & \\
\hline \multicolumn{4}{|c|}{${ }^{*}$ Data is presented as a number (percentage). } \\
\hline \multicolumn{4}{|l|}{$\unrhd$ Chi-square test was used. } \\
\hline \multicolumn{4}{|c|}{ T group =Patients received topical anesthesia } \\
\hline $\mathrm{TC}$ group $=$ Patients received & opical anesthesi & -crayotherapy & \\
\hline
\end{tabular}

Patients with sedation scores below four were given fentanyl at a dose of $1 \mu \mathrm{g} / \mathrm{kg}$. The relative frequency of patients who received fentanyl was not significantly different between the TC $(n=21$ [48.8\%]) and T $(n=22[51.2 \%])$ groups $(p=1.000)$. 
As shown in Fig. 2, the two groups were not significantly different in the hemodynamic evaluation, including heart rate $(p=0.347)$ and mean arterial pressure $(p=0.142)$.

No patient developed hypoxia, hypotension, bradycardia, excessive drowsiness (or sleepiness), and vomiting during the surgery. Table 5 shows that the postoperative side effects did not show a statistically significant difference between the two groups. Only one case of chills and one case of headache were recorded in the TC group. In the control group, we recorded three incidents of nausea and two cases of headache.

Table 5

Comparison of postoperative side effects in the two groups

\begin{tabular}{|lllll|}
\hline Variable & P-value & T group $(\mathbf{n = 4 0 )}$ & TC group $(\mathbf{n = 4 0 )}$ & P-value \\
\hline shivering & $2 / 0$ & $(0) 0$ & $(5 / 2) 1$ & $2 / 0$ \\
\hline headache & $9 / 0$ & $(5) 2$ & $(5 / 2) 1$ & $9 / 0$ \\
\hline nausea & $2 / 0$ & $(5 / 7) 3$ & 0 & $2 / 0$ \\
\hline *Data is displayed as a number (percentage). \\
\hline ■ Chi-square test was used. \\
\hline T group =Patients received topical anesthesia. \\
\hline
\end{tabular}

\section{Discussion}

The findings of our study revealed that cryotherapy is a complementary method with topical anesthesia and has a significant positive effect on pain intensity during surgery and surgeon satisfaction. This finding is partly consistent with a previous study by Gutierrez-Carmona FJ et al. ${ }^{[4]}$ which declared that the pain level in cryoanalgesia clear corneal phacoemulsification and topical anesthesia are almost equal. They suggested that cryoanalgesia is a suitable technique for anesthetic allergy cases. ${ }^{[4]}$

While, in the Coelho et al studies, which were performed on patients undergoing cataract surgery, no significant difference was observed in the amount of pain between the topical anesthesia versus topical combined to cryotherapy. ${ }^{[8]}$

However, it seems that methodological differences in the studies can explain some of these contradictions. For example, the number of surgeons, surgery on one or both eyes, different groups of patients in terms of age and sex, different pain sensitivity and perception of patients and different tools to assess the amount of pain and also the type of medication prescribed to reduce patients' anxiety. Furthermore, the old age of patients and the lack of accurate answers to the questionnaire can also be another reason for the discrepancies in the results. 
Nevertheless, it is known that cryotherapy having anti-inflammatory and analgesic effects. ${ }^{[6]}$ Cryotherapy reduces the conduction velocity of the nerve fibers due to the asynchronous transmission in pain fibers, the release of endorphins, and the inhibition of spinal neurons, and an increase in the refractory period, which leads to a gradual lessening in the transmission of impulses in the sensitive nerves. ${ }^{[11]}$ It has been suggested that the use of cold intraocular solution during the phacoemulsification, prevents the transmission of mechanical pain impulses continuously. ${ }^{[11]}$

Another mechanism suggested for the analgesic effects of cryotherapy is cold-induced vasoconstriction, which can reduce blood flow to the target tissue and reduce the release of inflammatory mediators, and also, it promotes the accumulation of local anesthetic in the target tissue. ${ }^{[6,11]}$ Finally, the synergistic effect of cryotherapy along with topical anesthesia led to a greater sense of comfort in patients during surgery.

The other notable finding is that the level of sedation of patients in both groups was not statistically significant. That's probably, all patients in the ward were given $3 \mathrm{mg}$ of melatonin one hour before entering the operating room.

In the same way, the patient satisfaction numbers (IOWA) were the simillar in both groups in our study.

Since the excessive sedation and decreased cognitive function leading to loss of patient cooperation with the surgeon, and also make the evaluation of patients' pain inaccurate. Thereby, to avoid bias, in the present study, one hour before entering the operating room, we used melatonin as an anxiolytic drug to increase patient satisfaction. In previous studies, the anxiolytic effect of melatonin without cognitive dysfunction was demonstrated as a pretreatment in various surgeries. ${ }^{[3]}$

Another finding of this study was the greater satisfaction of the surgeon in the cryoanalgesia group. The lower pain scores of patients during surgery in the TC group seemed to be directly related to the surgeon's satisfaction.

Although, our study had some limitations; old age of patients and low level of their cooperation to answer the questions of the inevitable questionnaire. We recommend future studies in patients with bilateral cataracts.

\section{Conclusion}

The findings of the present study revealed that cryotherapy as a complementary method with topical anesthesia could reduce patients' pain scores during surgery and enhance surgeon satisfaction.

\section{Declarations}

Ethics approval and consent to participate The use of data for this study was approved by Qazvin university of medical sciences with ID: IRQUMS.REC. 1396.12 


\section{Acknowledgement}

Authors of this paper are thankful for the valuable cooperation of staffs of Clinical Research Development Unit at hospitals of Velayat and Kosar in Qazvin for preparing this paper.

\section{Authors' contributions}

The first and corresponding author of this paper is Marzieh Beigom Khezri; Professor of Qazvin University of Medical Science, which responsible for design of study and preparing the manuscript. Abbas Akrami; Ophthalmolgist, refractive and cataract surgeon, Fellowship of Glaucoma; which responsible for conception and design of study. Matina Majdi ; Resident of Anesthesiology has done acquisition of data and drafting the manuscript. , Bijan Gahandideh; The nurse has done acquisition of data . Navid Mohammadi ; Professor of Community and preventive medicine who analyzed and interpreted the data and critically reviewed the manuscript.

\section{Funding}

This study supported by Vice Chancellor for Research, Qazvin University of Medical Sciences

\section{Author details}

${ }^{1}$ Metabolic Diseases Research Center, Research Institute for Prevention of Non-Communicable Diseases, Qazvin University of Medical Sciences, Qazvin, Iran. ${ }^{2}$ Department of Ophthalmology, Qazvin University of Medical Sciences, Qazvin, Iran. ${ }^{3}$ Resident of Anesthesiology, Qazvin University of Medical Sciences, Department of Anesthesiology. Qazvin, Iran. ${ }^{4}$ Department of Nursing. Qazvin University of Medical Sciences, Qazvin, Iran. ${ }^{5}$ Department of preventive and community medicine. Qazvin University of Medical Sciences, Qazvin, Iran.

\section{Availability of data and materials}

The data that support the findings of this study are available on request from the corresponding author

\section{Consent for publication}

The manuscript does not contain any individual person's data in any form.

\section{Competing interests}

There is not conflict of interest

\section{References}

1. Klein R, Klein BEK.The prevalence of age-related eye diseases and visual impairment in aging: current estimates. Invest Ophthalmol Vis Sci. 2013. 
2. Khanna R, Pujari S, Sangwan V. Cataract surgery in developing countries. Curr Opin Ophthalmol 2011;22(1):10-4.

3. Khezri M, Merate $\mathrm{H}$. The effects of melatonin on anxiety and pain scores of patients, intraocular pressure, and operating conditions during cataract surgery under topical anesthesia. Indian J Ophthalmol. 2013;61(7):319-24

4. Gutierrez-Carmona FJ, Alvarez-Marin J. Randomized comparative clinical study of cryoanalgesia versus topical anesthesia in clear corneal phacoemulsification. J Cataract Refract Surg 2005; 31:1187-1193.

5. Torres-Moreno AJ. Anestesiatopica con solucionesfr ıas en facoemulsificacion [Topical anesthesia with cold solutions in phacoemulsification]. Revista Mexicana de Oftalmologia 2009; 83(4):197200.

6. Silva DA, Peixot GFG, Rodrigues KMS, Farias VX, Analgesic efficacy of the association of cryotherapy and transcutaneous electrical nerve stimulation. BrJP 20181 no.3 São Paulo July/Sept. https://doi.org/10.5935/2595-0118.20180052

7. Lloyd J W, Barnard J D, Glynn C J. Cryoanalgesia. A new approach to pain relief. Lancet.1976; 30;2(7992):932-4.

8. Coelho RP, Biaggi RH, Jorge R, Rodrigues MdLV, Messias A. Clinical study of pain sensation during phacoemulsification with and without cryoanalgesia. Journal of Cataract \& Refractive Surgery. 2015 Apr 41(4):719-23.

9. Dexter F, Aker J, Will A. Development of a measure of patient satisfaction with monitored anesthesia care; the lowa satisfaction with anesthesia care. Anesthesiology. 1997;87:865-73.

10. Dexter F, Candiotti KA Multicenter assessment of the lowa Satisfaction with Anesthesia Scale, an instrument that measures patient satisfaction with monitored anesthesia care. Anesth Analg.2011; 113:364-368.

11. Nadler SF, Weingand K, Kruse RJ. The physiologic basis and clinical applications of cryotherapy and thermotherapy for the pain practitioner. Pain Physician. 2004;7(3):395-9.

\section{Tables}

Due to technical limitations, table 1-5 is only available as a download in the Supplemental Files section.

\section{Figures}




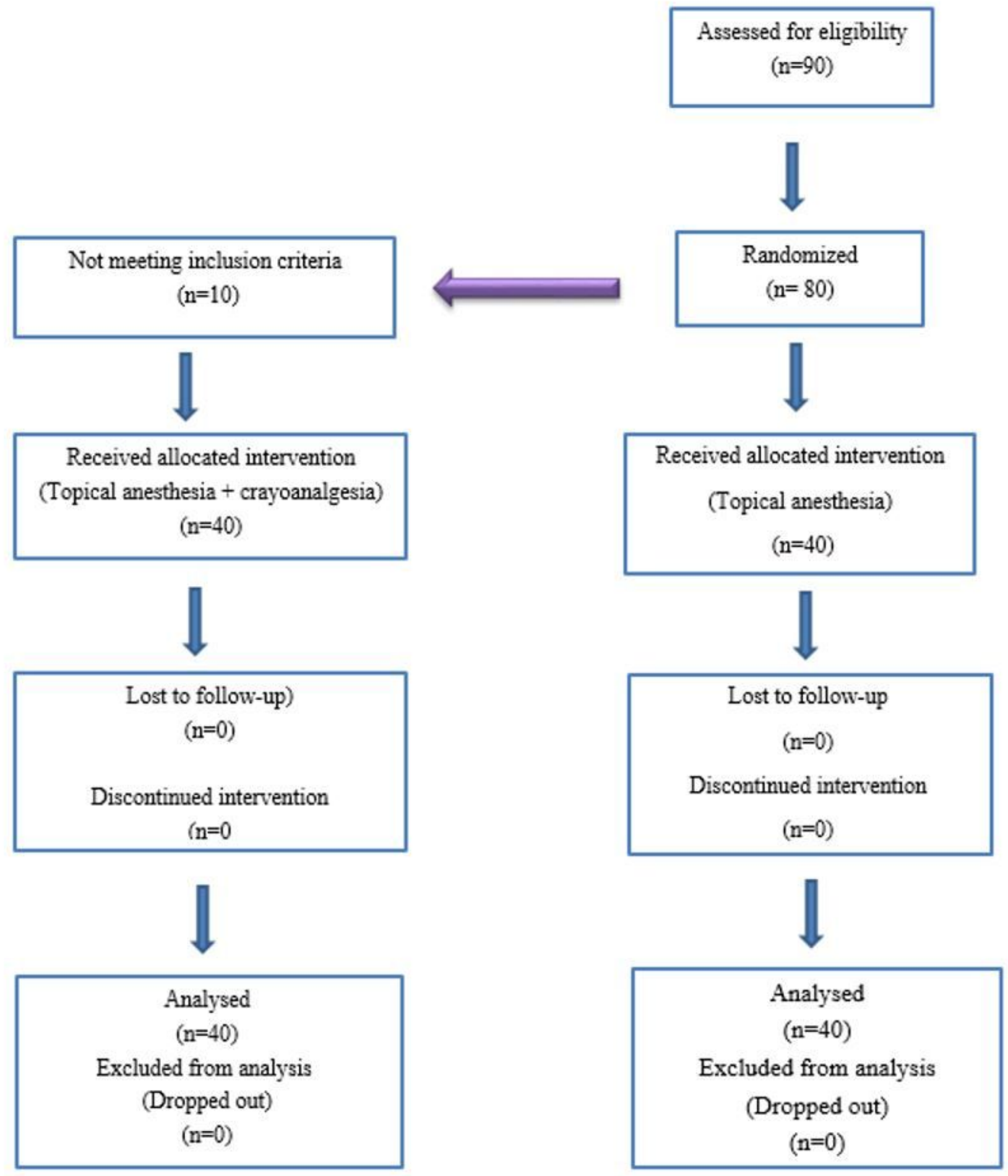

Figure 1

Consort flow diagram 


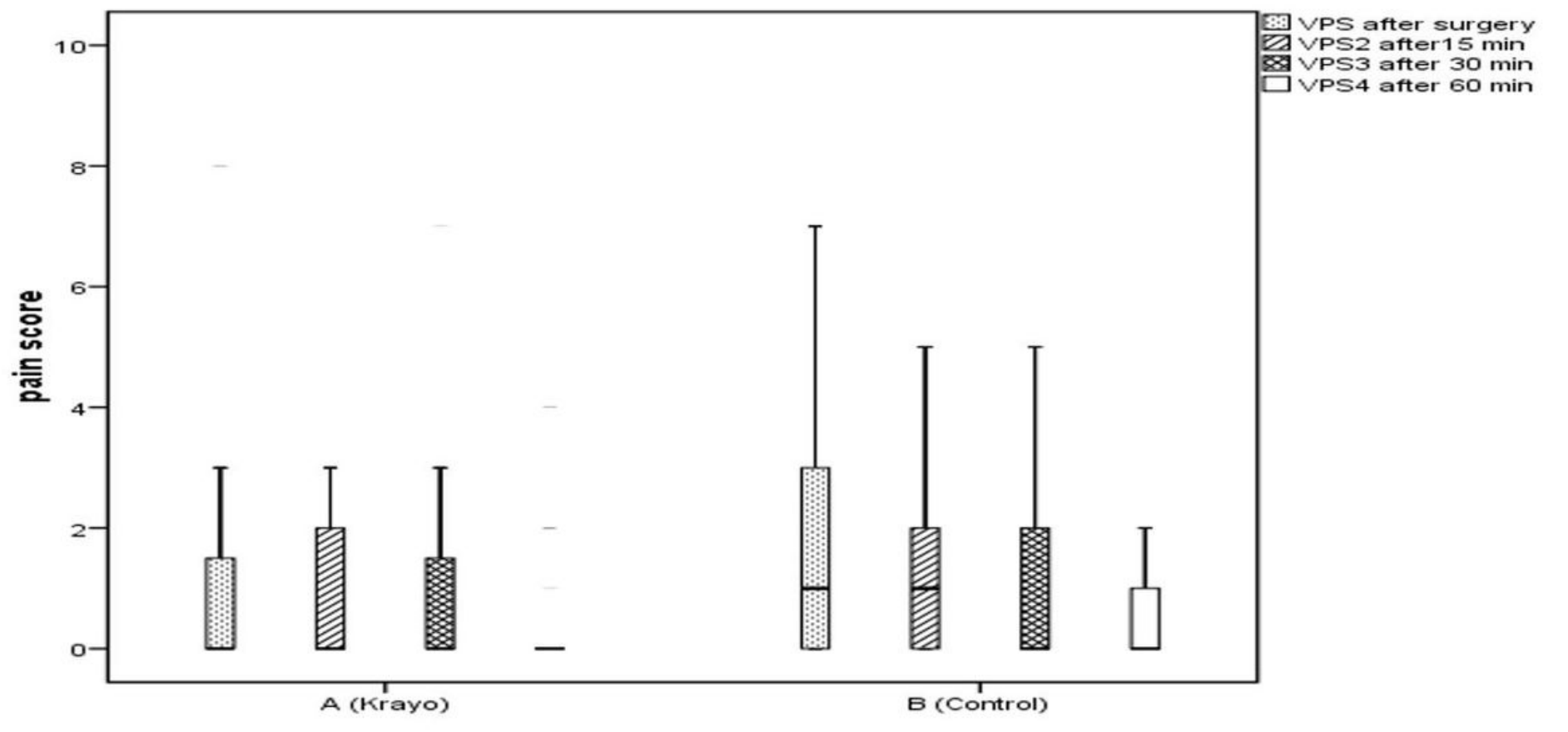

Figure 2

Intensity of pain in the two groups in $15,30,60$ minutes after surgery 

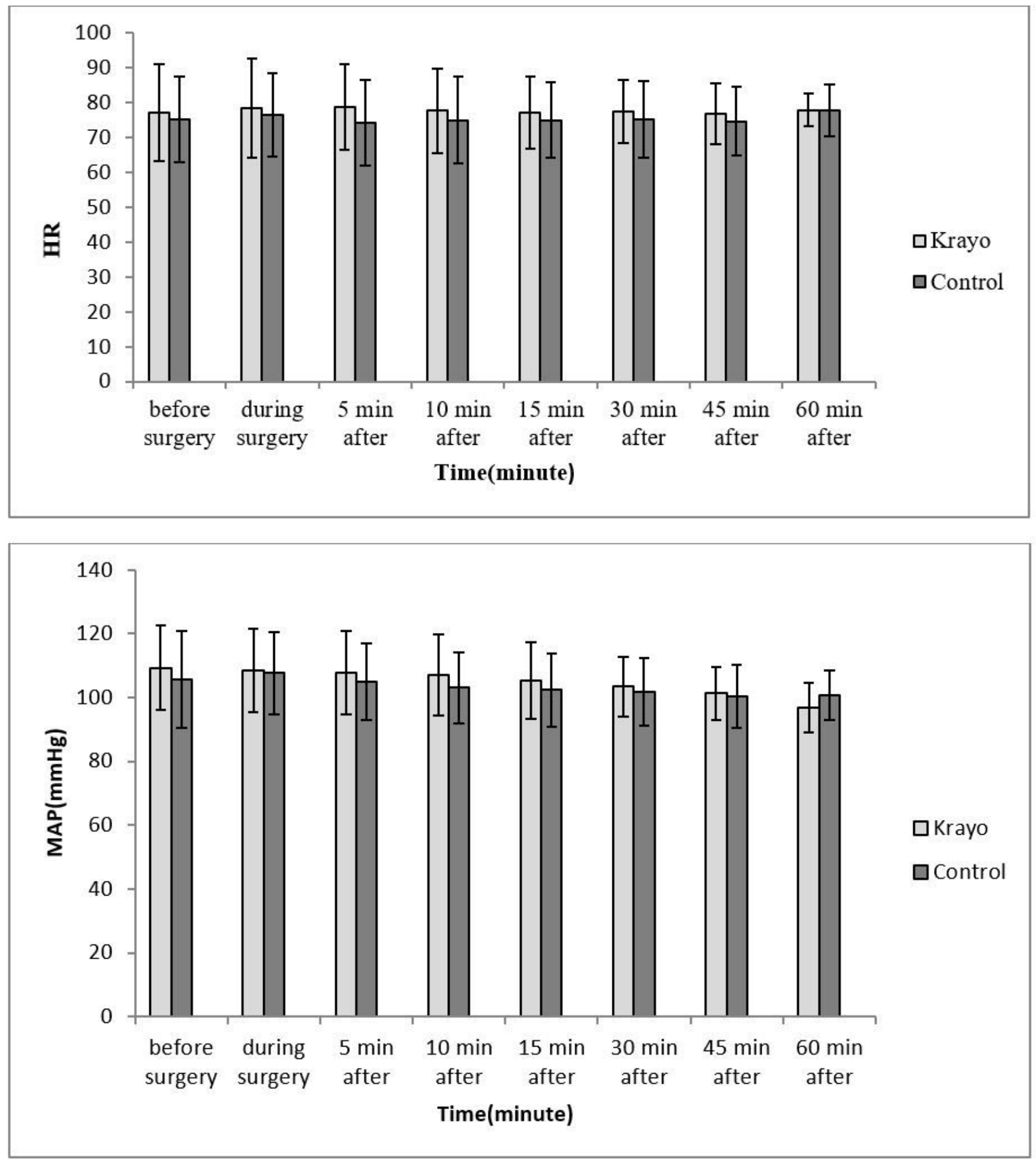

Figure 3

Hemodynamic variables.

\section{Supplementary Files}

This is a list of supplementary files associated with this preprint. Click to download. 
- Table1.jpg

- Table2.jpg

- Table3.jpg

- Table4.jpg

- Table5.jpg 